\title{
Avaliação da cobertura de vacinação em Teresina - Piauí (Brasil - 1983)
}

\author{
C.L. Szwarcwald* \\ J.G. Valente**
}

The objective of the present study was to estimate the immunization coverage of polio, triplice, BCG and measles in children one year of age in the urban area of the municipality of Teresina (Piaui), in 1983. Two sampling methods were used. Henderson and Sundaresan ${ }^{7}$ and an adaptation of the first and the results were later compared. An analysis was carried out to discriminate those children who had completed their vaccinations from those who had failed to do so, based on social indicators such as education of the parents and number of residents and children in each household. Part of the study focused on the principal motives given by the mother for not having vaccinated their children.

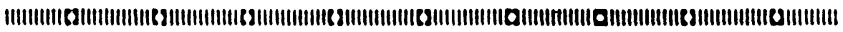

* Escola Nacional de Saúde Pública - FIOCRUZ - Rio de Janeiro

* Departamento de Vigilância Epidemiológica da Secretaria de Estado de Saúde e Higiene - Rio de Janeiro

\section{INTRODUÇÃO}

No Brasil, a estatística de mortalidade proporcional por doenças imunizáveis para o ano de 1980 ainda se encontra alta e em torno de $11 \%$ para crianças de 1 a 5 anos $^{4}$.

Em paralelo com estes dados, coberturas de vacinação são, em geral, mal-estimadas pelos serviços de saúde, desconhecendo-se o número real de crianças vacinadas. Além disso, baseando-se somente na avaliação administrativa dos serviços de saúde, fica difícil conhecer o número de crianças que completaram o quadro de imunização.

Para a implementação e avaliação de um programa de imunizações no país, é importante que se estimem coberturas de vacinação próximas da realidade. Para isto, devem ser utilizadas técnicas de amostragem que se mostrem eficientes a baixo custo. Henderson e Sundaresan ${ }^{7}$ propuseram um método de amostragem para avaliação de cobertura de imunizações que foi aplicado primeiramente nos Estados Unidos e depois em comunidades do Oeste da África.

Experimentos semelhantes ${ }^{2,6}$, no Brasil, já foram feitos em Araraquara (São Paulo) e Recife (Pernambuco). Um outro procedimento amostral foi sugerido por Manceau e aplicado em Salvador, Bahia ${ }^{1}$.

Neste trabalho pretendemos comparar duas metodologias de amostragem, a de Henderson e Sundaresan ${ }^{7}$ e uma modificação desta como proposta pelo Departamento de Epidemiologia e Métodos Quantitativos em Saúde da Escola 
Nacional de Saúde Pública. O presente estudo avaliou a cobertura de vacinação antipólio, DPT, BCG e anti-sarampo, em crianças de 12 a 23 meses de idade, na área urbana do município de Teresina (Piauí). Analisamos também alguns indicadores sociais que permitiram estabelecer diferenças entre os grupos de crianças que completaram e as que não completaram o quadro de vacinação.

Uma parte do nosso trabalho dirigiu-se para a identificação dos principais motivos apontados pelas mães por não terem completado a vacinação de suas crianças. $O$ conhecimento de tais motivos é fundamental para o bom termo de um programa de imunização.

\section{METODOLOGIA}

A área urbana do município de Teresina (Piauí) tem cerca de 350.000 habitantes e foi subdividida em 298 setores censitários pelo IBGE à época do censo (1980).

De acordo com o método preconizado por Henderson e Sundaresan ${ }^{7}$, trinta setores foram escolhidos com probabilidade proporcional ao número de habitantes. Selecionandose aleatoriamente um ponto inicial de percurso, os sete primeiros domicílios em que residissem crianças na faixa etária de 12 a 24 meses foram escolhidos para o inquérito.

$O$ segundo método aplicado constitui em modificar o primeiro na etapa da seleção dos 7 domicílios no setor censitário, com o intuito de diminuir o viés introduzido pelo fato das crianças estarem sendo selecionadas em conglomerados de 7 domicílios. Assim, o segundo método constitui em conduzir um pequeno censo em cada um dos 30 setores selecionados para identificação de todos os domicílios de cada setor que se prestavam ao inquérito, isto é, domicílios em que residissem pelo menos uma criança de 12 a 23 meses de idade. Após esta identificação, 7 domicílios foram escolhidos de maneira sistemática, de modo a cobrir todo o setor censitário.

Em ambos os métodos de amostragem, para cada criança selecionada foram preenchidas as fichas de informações sobre data de nascimento da criança, escolaridade do pai e da mãe, número de moradores, inclusive o número de crianças menores de 5 anos residentes no domicílio e ocorrência na criança, até a data da entrevista, de alguma das doenças preveníveis por imunização.

Maior destaque foi dado ao preenchimento do quadro de imunizações. Da caderneta de vacinas foram copiadas as datas de vacinas antipólio (3 doses e reforço), tríplice (3 doses e reforço), BCG e anti-sarampo. $\mathrm{Na}$ ausência da caderneta, informações orais eram marcadas com um código especial. No caso da BCG, os entrevistadores foram orientados para buscar a cicatriz na região deltóide do braço direito. As unidades de Saúde onde as crianças se vacinaram foram também anotadas para uma posterior confirmação das informa- 
ções orais. Datas de campanha de vacinação antipólio foram também coletadas quando presentes.

No caso do quadro de vacinação estar incompleto, a mãe ou informante especificava os motivos pelos quais tinha deixado de dar algumas vacinas aos seus filhos.

Coberturas de vacinação foram estimadas através de intervalos de confiança, segundo Cochran ${ }^{5}$, em amostragem por conglomerados. Os efeitos de desenho foram calculados para avaliação e comparação das duas metodologias de amostragem. A variância encontrada com a amostragem por conglomerados foi comparada com a variância esperada se 0 desenho fosse aleatório ${ }^{6}$. Para a diferenciação dos dois grupos de crianças, as que apresentavam quadro completo de vacinação daquelas com quadro incompleto, foram utilizados testes estatísticos usuais com $t$ de Student, $\chi^{2}$ de Pearson e a correlação de Kendall $(\zeta a)$.

O trabalho de campo desta avaliação foi feito pelos alunos do Curso Regionalizado de Saúde Pública do Estado do Piauí (num total de 29), com finalidade didática, como prática do Curso do Programa Ampliado de Imunizações (P.A.I.).

Cada aluno ficou responsável por realizar a avaliação de um setor censitário pelos dois métodos de amostragem. Pelo procedimento proposto por Henderson e Sundaresan ${ }^{7}$, que chamaremos procedimentos-OMS, um total de 205 crianças foi avaliado. Pelo outro procedimento, que denominaremos procedimento-SORTEIO, um total de 204.

\section{RESULTADOS}

As estimativas das coberturas de vacinação e seus respectivos intervalos a $95 \%$ de confiança encontram-se na Tabela 1 , onde são apresentadas, separadamente, as estimativas calculadas a partir de informações obtidas exclusivamente através de caderneta de vacinação e aquelas obtidas levando-se em conta, também, a informação verbal do responsável pela criança apesar da ausência de qualquer comprovação. No presente trabalho, foram avaliadas apenas as estimativas relativas à presença de comprovação através da caderneta de vacinação, por não ter sido possível avaliar o grau de confiabilidade das informações verbais prestadas pelos responsáveis. Dessa forma, verificamos que a maior cobertura é para BCG, seguida das três doses de antipólio, cerca de $60 \%$, enquanto que as coberturas de anti-sarampo e tríplice são baixas, por volta de $45 \%$.

Observamos que menos de $40 \%$ das crianças estão completamente imunizadas em Teresina *

$\mathrm{Na}$ Tabela 2, mostramos as estatísticas t de comparação das duas coberturas de vacinação comprovadas pelos dois procedimentos. Em nenhum caso o teste mostrou diferença significativa entre as proporções, ao nível de significância igual a $5 \%$.
" Chamamos de "Quadro completo" às crianças que possuam 3 doses de antipolio (rotina ou campanha), 3 doses de DPT e 1 dose de BCG e anti-sarampo 


\section{TABELA 1}

Coberturas de vacinação, Teresina 1983

\begin{tabular}{llccc}
\hline \multicolumn{1}{c}{ Vacina } & Procedimento & $\begin{array}{c}\text { Coberturas com } \\
\text { Caderneta \% }\end{array}$ & $\begin{array}{c}\text { Intervalos de } \\
\text { Confiança \% }\end{array}$ & $\begin{array}{c}\text { Coberturas Incluindo } \\
\text { Informaçōes Verbais \% }\end{array}$ \\
\hline Anti-sarampo & Sorteio & 48,04 & $41,31-54,77$ & 55,39 \\
& OMS & 40,98 & $33,19-48,76$ & 51,71 \\
BCG & Sorteio & 61,27 & $54,16-68,39$ & 69,12 \\
& OMS & 58,54 & $49,63-67,44$ & 64,88 \\
DPT (3 doses) & Sorteio & 46,57 & $39,57-53,56$ & 52,94 \\
& OMS & 43,41 & $35,01-51,82$ & 53,17 \\
Antipólio* & Sorteio & 57,35 & $50,21-64,49$ & 64,71 \\
(3 doses) & OMS & 53,17 & $43,63-62,71$ & 63,90 \\
Camp. Pólio & & 60,29 & $50,42-70,17$ & 60,29 \\
(2 doses ou mais) & Sorteio & 58,54 & $46,10-70,97$ & 58,54 \\
& OMS & 37,25 & $30,11-44,40$ & 42,65 \\
& Sorteio & 32,20 & $24,15-40,24$ & 42,44 \\
\hline
\end{tabular}

*Incluindo doses nas campanhas.

TABELA 2

Teste de Comparação das Proporções

\begin{tabular}{lcc}
\hline & $\mathrm{t}_{\text {calc }}$ & $\mathrm{P}$ \\
\hline BCG & 0,47 & NS \\
DPT & 0,57 & NS \\
Antipólio & 0,69 & NS \\
Anti-sarampo & 1,34 & NS \\
Quadro Completo & 0,92 & NS \\
Camp. Pólio & 0,22 & NS \\
\hline
\end{tabular}

Na Tabela 3, apresentamos os efeitos de desenho nos procedimentos OMS e SORTEIO. Verificamos que o procecimento-SORTEIO funciona como uma amostragem aleatória simples e que no procedimento-OMS o efeito de desenho não ultrapassa 2, como previsto ${ }^{7}$. Exceção só é encontrada na avaliação da cobertura das vacinas antipólio nas campanhas.

Para a diferenciação dos dois grupos de crianças, as que têm quadro completo de vacinação daquelas que o têm incompleto, foram utilizadas as crianças selecionadas pelo procedimento-SORTEIO. Identificamos que a variável mais discriminadora dos dois grupos é a escolaridade da mãe. Quanto menor o nível de instrução, maior é a proporção de crianças com falhas na imunização (Tabela 4). Para domicí- 
TABELA 3

Comparação dos dois procedimentos

\begin{tabular}{llcc}
\hline Procedimento & $\begin{array}{c}\text { Tamanho do } \\
\text { Intervalo } \\
\text { (P/ cada lado) }\end{array}$ & $\begin{array}{c}\text { Efeito } \\
\text { de } \\
\text { Desenho }\end{array}$ \\
\hline \multirow{2}{*}{ BCG } & Sorteio & 6,73 & 1,0 \\
DPT & OMS & 7,79 & 1,3 \\
& Sorteio & 7,00 & 1,0 \\
Antipólio & OMS & 8,40 & 1,5 \\
& Sorteio & 7,14 & 1,1 \\
Anti-sarampo & OMS & 9,54 & 1,9 \\
& Sorteio & 6,73 & 1,0 \\
Quadro Comple to & OMS & 7,79 & 1,3 \\
& OMS & 7,14 & 1,2 \\
Camp. Pólio & Sorteio & 8,05 & 1,6 \\
& OMS & 9,87 & 2,1 \\
\hline
\end{tabular}

TABELA 4

Situação do quadro de imunizações por escolaridade da mãe

\begin{tabular}{lcccc}
\hline $\begin{array}{l}\text { Escolaridade } \\
\text { Quadro }\end{array}$ & $\begin{array}{c}\text { Primário } \\
\text { Incompleto }\end{array}$ & $\begin{array}{c}\text { Primário } \\
\text { Completo }\end{array}$ & $\begin{array}{c}\text { 19 Grau } \\
\text { Incompleto }\end{array}$ & $\begin{array}{c}1 \text { 19 Grau } \\
\text { Completo }\end{array}$ \\
\hline Completo & 14 & 16 & 21 & 21 \\
& $(21 \%)$ & $(30 \%)$ & $(60 \%)$ & $(64 \%)$ \\
Incompleto & 53 & 37 & 14 & 12 \\
Total & $(79 \%)$ & $(70 \%)$ & $(40 \%)$ & $(36 \%)$ \\
\hline
\end{tabular}

$\mathrm{X}^{2}=26,01(\mathrm{p}<1 \%)$

$\zeta_{\mathrm{a}}=-0,38$

lios com grande número de moradores e com mais de 2 (duas) crianças menores de 5 anos, a proporção de crianças incompletamente vacinadas é significativamente maior (Tabelas 5 e 6). A idade das mães, no entanto, mostrou-se estatisticamente igual nos dois grupos (Tabela 7).

$\mathrm{Na}$ Tabela 8 apresentamos os motivos expostos pelas mães (ou responsáveis) por não terem completado a rotina de imunizações de suas crianças. O motivo mais apontado foi a presença de doença na criança (gripe, diarréia, etc.) na época prevista de vacinação. Muito indicada também foi a "impossibilidade de levar a criança ao posto". Além do motivo apontado também com algum peso "faltou vacina no posto", a maioria dos outros apresenta desconhecimento total ou parcial sobre a importância da imunização. 


\section{TABELA 5}

Situação do quadro de imunizaçঠes por número de moradores do domictio

\begin{tabular}{lccc}
\hline \multicolumn{1}{c}{ No de Moradores } & 2,3 ou 4 & 5,6 ou 7 & 8 ou mais \\
Quadro & & & \\
\hline Completo & $\begin{array}{c}20 \\
(43 \%)\end{array}$ & $\begin{array}{c}38 \\
(45 \%)\end{array}$ & $\begin{array}{c}15 \\
25 \%)\end{array}$ \\
Imcompleto & 27 & 47 & 46 \\
& $(57 \%)$ & $(55 \%)$ & $(75 \%)$ \\
Total & 47 & 85 & 61 \\
\hline $\mathrm{X}^{2}=6,70(5 \%>\mathrm{p}>1 \%)$ & & & \\
$\zeta_{\mathrm{a}}=0,16$ & &
\end{tabular}

TABELA 6

Situação do quadro de imunizaçōes por número de crianças menores de 5 anos no domic ilio

\begin{tabular}{lccc}
\hline \multicolumn{1}{c}{ No de Crianças } & 1 & 2 & 3 ou mais \\
Quadro & & & \\
\hline Completo & 33 & 30 & 10 \\
& $(45 \%)$ & $(43 \%)$ & $(20 \%)$ \\
Incompleto & 40 & 39 & 41 \\
Total & $(55 \%)$ & $(57 \%)$ & $(80 \%)$ \\
\hline $\mathrm{X}^{2}=9,83(\mathrm{p}<1 \%)$ & 73 & 69 & 51 \\
$\zeta_{\mathrm{a}}=0,20$ & & &
\end{tabular}

TABELA 7

Idade média da mãe nos dois grupos

\begin{tabular}{lrcc}
\hline \multicolumn{1}{c}{ Quadro } & $\mathrm{N}$ & $\overline{\mathrm{X}}$ & $\mathrm{S}$ \\
\hline Completo & 76 & 27,07 & 5,66 \\
Incompleto & 120 & 27,36 & 6,45 \\
\hline
\end{tabular}

$\mathrm{t}_{\mathrm{cal}}=0,32(\mathrm{NS})$

TABELA 8

Motivos expostos pelas mães

\begin{tabular}{ccc}
\hline Motivo & No & $\%$ \\
\hline $\begin{array}{c}\text { 1 - Doença da criança } \\
\text { - Impossibilidade de levar a } \\
\text { criança ao posto }\end{array}$ & 42 & 33 \\
& 16 & 13
\end{tabular}


TABELA 8 (continuação)

\begin{tabular}{lrr}
\hline \multicolumn{1}{c}{ Motivo } & No & $\%$ \\
\hline 3 - Não sabia que estava incompleto & 13 & 10 \\
4 - Displicência dos pais & 11 & 9 \\
5 - Falta de vacina no posto & 11 & 9 \\
6 - Achava que já tinha vacinado & 9 & 7 \\
na campanha & 6 & 5 \\
7 - Descrédito na vacina & 5 & 4 \\
8 - Medo ou resistência & 5 & 4 \\
9 - Ignorância total sobre vacinação & 5 & 4 \\
10 - Acesso difícil ao posto & 3 & 2 \\
11 - Mal atendimento no posto & & \\
\hline
\end{tabular}

\section{COMENTÁRIOS}

No Brasil, desde 1980, vêm se realizando duas vezes ao ano dias nacionais de imunização contra a poliomielite. As crianças que participaram deste estudo deveriam ter recebido, portanto, pelo menos duas doses de vacina, respectivamente, em junho e agosto de 1982. Encontramos que apenas $60 \%$ das crianças amostradas tinham recebido duas ou mais doses de vacina, o que nos pareceu uma cobertura muito abaixo da esperada, apesar de, neste caso, ter de se levar em conta o efeito de memória do responsável, já que as vacinas dadas na campanha nem sempre são anotadas na cardeneta.

Com os resultados apresentados para as coberturas de vacinação, podemos concluir que o nosso método estima proporções equivalentemente a uma amostra aleatória simples, enquanto que, com o método de Henderson e Sundaresan ?, os intervalos de confiança não ultrapassam os $10 \%$ previstos para cada lado. Podemos discutir, então, as vantagens de cada método: se, por um lado, o procedimento-SORTEIO apresenta maior precisão com o mesmo tamanho de amostra, por outro ele apresenta maior dificuldade de aplicação que o procedimento-OMS, já que requer um censo prévio de cada um dos 30 setores. Tais dificuldades devem ser levadas em consideração numa próxima avaliação de cobertura em outra localidade do país.

A justificativa apresentada pelas mães para não terem concluído a vacinação de seus filhos pode ajudar a elucidar problemas encontrados na prática de imunizações. Concluise, como na investigação realizada no Suriname ${ }^{3}$, que uma das causas mais apontadas foi o fato da criança estar doente (apresentando gripe, diarréia, etc.) à época prevista para aplicação da vacina. Tendo em vista que crianças menores de 1 ano apresentam constantemente fezes líquidas e resfriados, deve-se procurar saber qual a orientação exata que está sendo dada às mães pelos serviços de saúde. Um ponto para investigação é saber se a orientação de não vacinar a 
criança por ela estar doente parte dos serviços de saúde ou de um conceito social próprio da comunidade. De qualquer forma, esta questão deve ser trabalhada na população, já que estudos realizados em crianças doentes e mal-nutridas, para avaliação dos benefícios e riscos de imunizações, resultaram em indicações favoráveis à aplicação das vacinas ${ }^{8}$.

Ainda gostaríamos de comentar sobre a importância da divulgação de um programa de imunizações, a nível de compreensão direta pela população de mais baixa renda e escolaridade. Se levarmos em conta que mais de $30 \%$ das crianças não têm seu quadro de imunizações completo por falta de informações, descrédito, medo ou não valorização da vacina por parte de seus pais ou responsáveis, concluiremos que grande ênfase deve ser dada a práticas de educação em saúde, relevando a importância da imunização, assim como à orientação com explicações detalhadas de datas de retorno aos postos de saúde para todas as vacinas ainda ausentes na criança.

\section{AGRADECIMENTOS}

Este trabalho foi realizado com o apoio do Curso Regionalizado de Saúde Pública do Piauí - Convênio Escola Nacional de Saúde Pública - FIOCRUZ/Secretaria Estadual de Saúde do Piauí, e do grupo do Programa Ampliado de Imunizações da Escola Nacional de Saúde Pública.

Agradecemos particularmente aos alunos do Curso Regionalizado de Saúde Pública do Piauí pela realização do trabalho de campo, à Prof a Maria Abigail Barbosa, pela supervisão da coleta dos dados, e à Profa Maria Francisca Duailibe Mascarenhas Barbosa, coordenadora do Curso, pelo grande apoio dado a esta investigação.

Agradecemos, ainda, as valiosas contribuições do Prof? Paulo Sabroza, do Departamento de Epidemiologia e Métodos Quantitativos em Saúde - ENSP, e da Drạ Itamara Meilman, coordenadora da Comissão de Recursos Humanos da Secretaria de Saúde do Município do Rio de Janeiro.

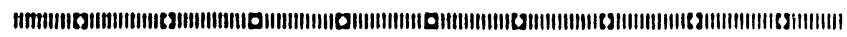

$O$ presente estudo avaliou a cobertura de vacinação antipólio, DPT, BCG e anti-sarampo em crianças de um ano de idade, na área urbana do municipio de Teresina (Piaui), em 1983. Dois métodos de amostragem, o de Henderson e Sundaresan ${ }^{7}$ e uma modificação deste foram utilizados posteriormente comparados quanto aos resultados. $A$ análise de alguns indicadores sociais, como escolaridade dos pais e número de moradores e de crianças do domicilio, nos permitiu estabelecer diferenças entre os grupos de crianças que completaram e as que não completaram $o$ quadro de imunizações. Uma parte do trabalho dirigiu-se 
para a identificação dos principais motivos apontados pelas mães por não terem completado a vacinação de suas crianças.

\section{REFERÊNCIAS BIBLIOGRÁFICAS}

1. BAHIA. Secretaria de Saúde. Informes sobre a avaliaçāo do programa de imunização do estado da Bahia. Salvador, 1983.

2. BOLETIN INFORMATIVO PAI. v. 2, n. 3, 1980.

3. BOLETIN INFORMATIVO PAI. v. 5, n. 2, 1983.

4. BRASIL Ministério da Saúde. Secretaria Nacional de Ações Básicas de Saúde. Estatisticas de mortalidade; Brasil, 1980. Brasília, Centro de Documentação do Ministério da Saú de, 1983.

5. COCHRAN, W.G. Sampling techniques. 2. ed. NewYork, John Wiley \& Sons, 1963.

6. CUNHA, C.C.L. de et alii. Avaliação de cobertura vacinal em Araraquara (SP). São Paulo, Faculdade de Saúde Pública, 1982.

7. HENDERSON, R.H. \& SUNDARESAN, T. Cluster sampling to asess immunization coverage; a review of experience with a simplified sampling method. Bull. WHO, 60 (2) :253-60, 1982.

8. WORLD HEALTH ORGANIZATION. Immunization of children; indications and contraindications for vaccines used in the expanded programme on immunization. EPI/GAG/82/WP. 8/ rev. 3. 